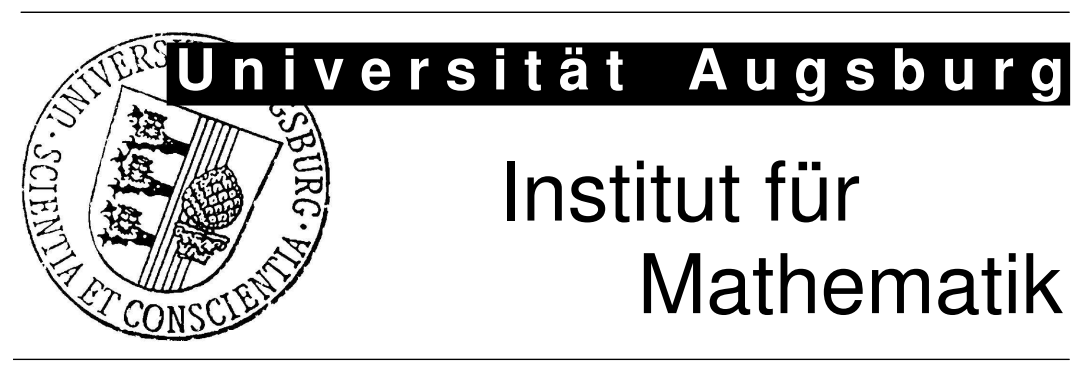

Alexandra Gaevskaya, Ronald H.W. Hoppe, Sergey Repin

Functional Approach to A Posteriori Error Estimation for Elliptic Optimal Control Problems with Distributed Control 


\section{Impressum:}

Herausgeber:

Institut für Mathematik

Universität Augsburg

86135 Augsburg

http://www . math. uni-augsburg.de/forschung/preprint/

ViSdP:

Ronald H.W. Hoppe

Institut für Mathematik

Universität Augsburg

86135 Augsburg

Preprint: Sämtliche Rechte verbleiben den Autoren (C) 2007 


\title{
FUNCTIONAL APPROACH TO A POSTERIORI ERROR ESTIMATION FOR ELLIPTIC OPTIMAL CONTROL PROBLEMS WITH DISTRIBUTED CONTROL
}

\author{
A. GAEVSKAYA *, R.H.W. HOPPE ${ }^{\dagger}$, AND S. REPIN $\ddagger$
}

\begin{abstract}
We present a new approach to the a posteriori analysis of distributed optimal control problems. It is based on functional type a posteriori estimates that provide computable and guaranteed bounds of errors for any conforming approximations of a boundary value problem. We derive computable two-sided a posteriori estimates for the cost functional and estimates for the approximations of state and control functions. Numerical results illustrate efficiency of the approach suggested.
\end{abstract}

AMS subject classifications. 65F10, 65N30

1. Introduction. During the past decade, the a posteriori error analysis of finite element approximations of boundary and initial-boundary value problems for partial differential equations and systems thereof has reached some state of maturity as documented by a series of monographs on this subject (cf., e.g., [1, 4, 5, 6, 7, 14, 19, 20, 32]). The a posteriori error analysis is usually based on a posteriori error estimates of the global discretization error or goal oriented error functionals. They provide an upper bound and sometimes also a lower bound of the overall error and serve as an indicator of the error distribution. There are several well-known approaches to a posteriori error control. Residual type estimators rely on the evaluation of the negative norm of the residual and can be traced back to the early work [2,3], whereas hierarchical type estimators locally approximate the error equation by higher order finite elements [6]. Another approach relies on local averaging. First suggested in [34, 35], it has been mathematically justified for superconvergent approximations (see, e.g.,[33]). A posteriori error indicators of this type have been further investigated by many authors including new variants of post-processing (averaging) of approximate solutions (see, e.g., [10] and the references therein). Finally, the so-called goal-oriented approach allows the consideration of rather general error functionals and extracts the error information from the associated dual problem (cf., e.g., $[5,16])$.

We further note that a convergence analysis of adaptive finite element methods in the sense of a guaranteed error reduction has been recently provided in [15] and $[26,27]$ for standard Lagrangian type finite element approximations of second order elliptic boundary value problems, whereas mixed and nonconforming finite elements as well as edge element approximations of Maxwell's equations have been considered in $[11,12]$ and $[13]$.

During the past couple of years, significant research efforts have been devoted to the development of adaptive finite element techniques for the numerical solution of optimal control problems governed by partial differential equations. We refer to $[8,9]$

\footnotetext{
*Institute of Mathematics, Universität Augsburg, D-86159 Augsburg, Germany

${ }^{\dagger}$ Department of Mathematics, University of Houston, Houston, TX 77204-3008, USA, and Institute of Mathematics, Universität Augsburg, D-86159 Augsburg, Germany

${ }^{\ddagger}$ St. Petersburg Department of V.A. Steklov Institute of Mathematics of Russian Academy of Sciences, 191023, St. Petersburg, Russia

${ }^{0}$ The work of the first and the third authors has been partially supported by Russian Foundation for Basic Researches under Grant No. 06-01-00641-a.

${ }^{0}$ The work of the second author has been partially supported by the NSF under Grant No. DMS-0411403.
} 
for unconstrained problems and to $[18,21,23,24,25]$ in case of control constraints.

In $[29,30,31]$, a posteriori error estimates of another type have been suggested. They have been derived by purely functional methods. Hence, they are applicable to any conforming approximation of a boundary-value problem under consideration. The estimates contain no mesh-dependent constants and provide a guaranteed upper bound for the difference between the exact solution and its approximation. Such functional type a posteriori estimates have been analyzed and numerically tested for a wide spectrum of linear and nonlinear problems (see, e.g., [28] and the references therein).

In this paper, we suggest a new approach to the a posteriori analysis of optimal control problems based upon such functional a posteriori error estimates and derive guaranteed and computable upper bounds for the cost functionals of distributed optimal control problems.

2. Problem statement and optimality conditions. We focus our attention on the following optimal control problem considered in a Lipschitz domain $\Omega \in \mathbb{R}^{n}$ with boundary $\Gamma:=\partial \Omega$.

Problem P. Given $y^{d} \in Y_{0}:=H_{0}^{1}(\Omega), u^{d} \in \mathcal{U}:=L_{2}(\Omega), f \in \mathcal{U}$, and $a>0$, consider the distributed control problem

$$
\begin{aligned}
\text { Minimize } & J(y, u):=\frac{1}{2}\left\|\nabla\left(y-y^{d}\right)\right\|^{2}+\frac{a}{2}\left\|u-u^{d}\right\|^{2} \\
\text { over } & (y, u) \in Y_{0} \times \mathcal{U}, \\
\text { subject to } & -\Delta y=u+f \quad \text { a.e. in } \Omega .
\end{aligned}
$$

Here and in the sequel, we adopt standard notation for Lebesgue and Sobolev spaces and norms.

By definition, $y_{g}$ is the unique solution of

$$
\begin{array}{rc}
-\Delta y_{g}=g & \text { in } \Omega, \\
y_{g}=0 & \text { on } \Gamma
\end{array}
$$

for $g \in \mathcal{U}$. Let $\left(y_{\bar{u}+f}, \bar{u}\right)$ be the exact solution of Problem P. To simplify the notation, we write $\bar{y}:=y_{\bar{u}+f}$.

TheOREM 2.1. Under the assumptions made Problem $P$ has a unique solution $(\bar{y}, \bar{u})$.

Proof. See [22].

It is not difficult to prove (see, e.g., [22]) that the solution satisfies the system of necessary conditions

$$
\begin{aligned}
& -\Delta \bar{y}=\bar{u}+f, \quad \bar{y} \in Y_{0}, \\
& \bar{u}=u^{d}+\frac{1}{a}\left(y^{d}-\bar{y}\right) .
\end{aligned}
$$

Remark 2.1. If $y^{d} \notin Y_{0}$, then the optimization problem can be still reduced to the above considered case. Indeed, let $\widehat{y}^{d}$ be a projection of $y^{d}$ on $Y_{0}$, i.e.,

$$
\int_{\Omega}\left(\nabla \widehat{y}^{d}-\nabla y^{d}\right) \cdot \nabla \psi d x=0, \quad \forall \psi \in Y_{0},
$$


then, $\left\|\nabla y-\nabla y^{d}\right\|^{2}=\left\|\nabla y-\nabla \widehat{y}^{d}\right\|^{2}+\left\|\nabla \widehat{y}^{d}-\nabla y^{d}\right\|^{2}$, and therefore,

$$
J(y, u)=\frac{1}{2}\left\|\nabla y-\nabla \widehat{y}^{d}\right\|^{2}+\frac{a}{2}\left\|u-u^{d}\right\|^{2}+c,
$$

where $c=\left\|\nabla \widehat{y}^{d}-\nabla y^{d}\right\|^{2}$ is a distance from $y^{d}$ to the set $Y_{0}$. Thus, the problem with functional

$$
\widehat{J}(y, u)=\frac{1}{2}\left\|\nabla y-\nabla \widehat{y}^{d}\right\|^{2}+\frac{a}{2}\left\|u-u^{d}\right\|^{2}
$$

has the same solution and the only difference consists of that the optimal value of the original functional exceeds the optimal value of $\widehat{J}(y, u)$ by the quantity $c$.

3. Basic problem and its transformation. In our further analysis, it is convenient to represent the basic problem in a somewhat different form, which, however, is equivalent to the original formulation. For an arbitrary function $\eta \in Y_{0}$, consider the problem

Problem $\mathbf{P}_{\eta}$. Given $\eta \in Y_{0}, y^{d} \in Y_{0}, u^{d} \in \mathcal{U}, f \in \mathcal{U}$, and $a>0$, consider the distributed control problem

$$
\begin{aligned}
\text { Minimize } & J_{\eta}(y, u):=\frac{1}{2}\|\nabla(y-\eta)\|^{2}+\frac{a}{2}\|u-\xi(\eta)\|^{2} \\
\text { over } & (y, u) \in Y_{0} \times \mathcal{U}, \\
\text { subject to } & -\Delta y=u+f \quad \text { a.e. in } \Omega .
\end{aligned}
$$

where $\xi(\eta)=u^{d}+\frac{1}{a}\left(y^{d}-\eta\right)$.

Proposition 3.1. For any $\eta \in Y_{0}$, there holds the following relation between cost functionals of Problem $P$ and Problem $P_{\eta}$ :

$$
J(y, u)=J_{\eta}(y, u)+C_{\eta},
$$

where

$$
\begin{aligned}
C_{\eta}: & =C\left(\eta ; y^{d}, f, u^{d}\right)=\frac{1}{2}\left\|\nabla \eta-\nabla y^{d}\right\|^{2}+\frac{a}{2}\left\|\xi(\eta)-u^{d}\right\|^{2}+ \\
& +\left(\int_{\Omega} \nabla \eta \cdot \nabla\left(y^{d}-\eta\right) d x-\int_{\Omega}(\xi(\eta)+f)\left(y^{d}-\eta\right) d x\right) .
\end{aligned}
$$

Proof. First, we rewrite the cost functional

$$
J(y, u)=\underbrace{\frac{1}{2}\left\|\nabla y-\nabla y^{d}\right\|^{2}}_{(I)}+\frac{a}{2}\left\|u-u^{d}\right\|^{2}
$$

as follows:

$$
\begin{gathered}
(I)=\frac{1}{2}\left\|\nabla y-\nabla y^{d}\right\|^{2}=\frac{1}{2}\left\|\nabla y-\nabla \eta+\nabla \eta-\nabla y^{d}\right\|^{2}= \\
=\frac{1}{2}\|\nabla y-\nabla \eta\|^{2}+\int_{\Omega} \nabla(y-\eta) \cdot \nabla\left(\eta-y^{d}\right) d x+\frac{1}{2}\left\|\nabla \eta-\nabla y^{d}\right\|^{2}= \\
=\frac{1}{2}\|\nabla y-\nabla \eta\|^{2}+\int_{\Omega} u\left(\eta-y^{d}\right) d x+C_{1}\left(\eta ; y^{d}, f\right),
\end{gathered}
$$


where $\eta$ is an arbitrary function in $Y_{0}$ and

$$
C_{1}\left(\eta ; y^{d}, f\right)=\frac{1}{2}\left\|\nabla \eta-\nabla y^{d}\right\|^{2}+\int_{\Omega} \nabla \eta \cdot \nabla\left(y^{d}-\eta\right) d x-\int_{\Omega} f\left(y^{d}-\eta\right) d x .
$$

Thus,

$$
J(y, u)=\frac{1}{2}\|\nabla(y-\eta)\|^{2}+\underbrace{\frac{a}{2}\left\|u-u^{d}\right\|^{2}+\int_{\Omega} u\left(\eta-y^{d}\right) d x}_{(I I)}+C_{1}\left(\eta ; y^{d}, f\right) .
$$

For the complex $(I I)$ we have

$$
\begin{aligned}
& (I I)=\frac{a}{2}\left\|u-u^{d}\right\|^{2}+\int_{\Omega} u\left(\eta-y^{d}\right) d x=\frac{a}{2}\|u\|^{2}-a \int_{\Omega} u \xi(\eta) d x+\frac{a}{2}\left\|u^{d}\right\|^{2}= \\
& =\frac{a}{2}\|u-\xi(\eta)\|^{2}+C_{2}\left(\eta ; y^{d}, u^{d}\right),
\end{aligned}
$$

where

$$
\begin{aligned}
C_{2}\left(\eta ; y^{d}, u^{d}\right) & =\frac{a}{2}\left\|u^{d}\right\|^{2}-\frac{a}{2}\|\xi(\eta)\|^{2}=\frac{a}{2}\left\|u^{d}-\xi(\eta)\right\|^{2}-a\|\xi(\eta)\|^{2}+a \int_{\Omega} u^{d} \xi(\eta) d x= \\
& =\frac{a}{2}\left\|u^{d}-\xi(\eta)\right\|^{2}-\int_{\Omega} \xi(\eta)\left(y^{d}-\eta\right) d x .
\end{aligned}
$$

Hence,

$$
J(y, u)=\frac{1}{2}\|\nabla(y-\eta)\|^{2}+\frac{a}{2}\|u-\xi(\eta)\|^{2}+\mathbf{C}_{\eta},
$$

where

$$
\mathbf{C}_{\eta}=C_{1}\left(\eta ; y^{d}, f\right)+C_{2}\left(\eta ; y^{d}, u^{d}\right)
$$

and we arrive at (3.3).

Corollary 3.2. Since the state equation (3.2) is one for the whole set of problems with $\eta$ and $\xi(\eta)$, we conclude that for any $\eta \in Y_{0}$, two problems - Problem $P$ and Problem $P_{\eta}$ - have one and the same solution $(\bar{y}, \bar{u})$.

Remark 3.1. We observe that

(a) if $\eta=y^{d}$, then $\xi(\eta)=u^{d}, \mathbf{C}_{\eta}=0$, and $J_{\eta}(y, u)=J(y, u)$;

(b) if $\eta=\bar{y}$, then $\xi(\eta)=\bar{u}, \mathbf{C}_{\eta}=J(\bar{y}, \bar{u})$, and $J_{\eta}(\bar{y}, \bar{u})=0$.

4. Two-sided estimates for the cost functional. In this section, we derive upper and lower estimates for the optimal value of the cost functional of the original problem $J(\bar{y}, \bar{u})$ using the results that were obtained in section 3 .

4.1. Upper estimates for the cost functional. Assume that $u \in \mathcal{U}$ is an admissible control function computed by a numerical procedure and $y_{u+f} \in Y_{0}$ is the respective state function. We do not know $y_{u+f}$ exactly and instead must operate with a certain approximation $y \in Y_{0}$. 
In view of Corollary 3.2, we can consider $(y, u)$ as an approximation of optimal control problem $P_{\eta}$ for any $\eta \in Y_{0}$. If we set $\eta=y$, then the expression of the cost functional comes in the form

$$
J_{y}\left(y_{u+f}, u\right)=\frac{1}{2}\left\|\nabla\left(y_{u+f}-y\right)\right\|^{2}+\frac{a}{2}\|u-\xi(y)\|^{2},
$$

where the first term represents the error of the approximate solution $y$ measured in the energy norm and, therefore, can be explicitly estimated by an a posteriori estimate. Since our goal is to obtain a guaranteed upper bound for the cost functional, we should apply functional estimates that provide computable and guaranteed upper bounds for the approximation errors of the boundary-value problem (2.2). As it is shown in [30], such estimates are of the form

$$
\left\|\nabla\left(y_{u+f}-y\right)\right\| \leq\|\tau-\nabla y\|+C_{\Omega}\|\operatorname{div} \tau+u+f\|,
$$

where $\tau$ is an arbitrary function in $H_{\text {div }}:=H(\Omega$, div $)=\left\{q \in L_{2}\left(\Omega ; \mathbb{R}^{n}\right) \mid \operatorname{div} q \in\right.$ $\left.L_{2}(\Omega)\right\}$, and $C_{\Omega}$ is the constant in the Friedrichs inequality

$$
\|v\| \leq C_{\Omega}\|\nabla v\|, \quad v \in Y_{0}
$$

for the domain $\Omega$. Mathematical justifications of functional type a posteriori estimates and their analysis can be found in the above cited literature. Here, we only recall the main properties of such estimates:

- for any approximation $y \in Y_{0}$, the right-hand side of (4.2) gives an upper bound of the error in the natural energy norm of the problem under consideration;

- its value is equal to zero if and only if $y$ coincides with $y_{u+f}$ and $\tau=\nabla y_{u+f}$;

- the estimate is consistent in the sense that its value tends to zero for any sequences $\left\{y_{k}\right\}$ and $\left\{\tau_{k}\right\}$ converging to the exact solution $y_{u+f}$ and its gradient $\nabla y_{u+f}$, respectively;

- the estimate is exact in the sense that there exists a function $\tau$ such that the inequality holds as an equality;

- the estimate does not depend on the mesh parameters and contains only one global constant.

Proposition 4.1. For the cost functional of Problem $P_{y}$ there holds the following upper estimate:

$$
J_{y}\left(y_{u+f}, u\right) \leq J_{y}^{\oplus}(u ; \beta ; \tau), \quad \forall \tau \in H_{\mathrm{div}}, \beta>0,
$$

where

$$
\begin{aligned}
J_{y}^{\oplus}(u ; \beta ; \tau) & =\frac{(1+\beta)}{2}\|\tau-\nabla y\|^{2}+\frac{(1+\beta)}{2 \beta} C_{\Omega}^{2}\|\operatorname{div} \tau+u+f\|^{2}+ \\
& +\frac{a}{2}\|u-\xi(y)\|^{2} .
\end{aligned}
$$

Proof. In view of (4.2), we obtain

$$
J_{y}\left(y_{u+f}, u\right) \leq \frac{1}{2}\left(\|\tau-\nabla y\|+C_{\Omega}\|\operatorname{div} \tau+u+f\|\right)^{2}+\frac{a}{2}\|u-\xi(y)\|^{2} .
$$

It easy to see that (4.3) follows if we estimate the first term of the right-hand side with the help of algebraic Young's inequality. $\square$ 
Corollary 4.2. For the cost functional of Problem $P$ the following inequality holds:

$$
J\left(y_{u+f}, u\right) \leq J^{\oplus}(y, u ; \beta ; \tau), \quad \forall \tau \in H_{\text {div }}, \beta>0,
$$

where

$$
J^{\oplus}(y, u ; \beta ; \tau)=J_{y}^{\oplus}(u ; \beta ; \tau)+C_{y} .
$$

Hereafter, we call $J^{\oplus}(y, u ; \beta ; \tau)$ the majorant of the cost functional. Another form of the majorant in [17].

4.2. Lower estimates for the cost functional. There exists an estimate that provides guaranteed and computable lower bound of error for conforming approximations of the state equation. It is given by the relation

$$
\frac{1}{2}\left\|\nabla\left(y_{u+f}-y\right)\right\|^{2} \geq-\int_{\Omega}\left(\frac{1}{2}|\nabla w|^{2}-\nabla w \cdot \nabla y+(u+f) w\right) d x, \forall w \in Y_{0}
$$

A justification of this estimate is presented in [28], where it is also shown that the estimate is exact in the sense that there exists $w \in Y_{0}\left(w=y-y_{u+f}\right)$ such that the inequality holds as an equality.

Substituting (4.5) into (4.1), we obtain a lower estimate for the cost functional, which does not contain $y_{u+f}$ :

$$
J_{y}\left(y_{u+f}, u\right) \geq-\int_{\Omega}\left(\frac{1}{2}|\nabla w|^{2}-\nabla w \cdot \nabla y+(u+f) w\right) d x+\frac{a}{2}\|u-\xi(y)\|^{2} .
$$

This inequality holds for any $u \in \mathcal{U}$. Therefore,

$$
\begin{aligned}
& J_{y}(\bar{y}, \bar{u})=\inf _{u \in \mathcal{U}} J_{y}\left(y_{u+f}, u\right) \geq \\
& \geq \inf _{u \in \mathcal{U}}\left\{-\int_{\Omega}\left(\frac{1}{2}|\nabla w|^{2}-\nabla w \cdot \nabla y+(u+f) w\right) d x+\frac{a}{2}\|u-\xi(y)\|^{2}\right\} .
\end{aligned}
$$

It is easy to observe that the minimizer is $u=\xi(y)+a^{-1} w$. Hence, we arrive at the following result.

Proposition 4.3. For the cost functional of Problem $P_{y}$ there holds the following lower estimate:

$$
J_{y}(\bar{y}, \bar{u}) \geq J_{y}^{\ominus}(w), \quad \forall w \in Y_{0}
$$

where

$$
\begin{aligned}
J_{y}^{\ominus}(w)= & -\frac{1}{2}\left(\|\nabla w\|^{2}+\frac{1}{a}\|w\|^{2}\right)-\int_{\Omega}(\xi(y)+f) w d x+ \\
& +\int_{\Omega} \nabla w \cdot \nabla y d x .
\end{aligned}
$$


Corollary 4.4. For the cost functional of Problem $P$ the following inequality holds:

$$
J(\bar{y}, \bar{u}) \geq J^{\ominus}(y ; w), \quad \forall w \in Y_{0},
$$

where

$$
J^{\ominus}(y ; w)=J_{y}^{\ominus}(w)+\boldsymbol{C}_{y} .
$$

Hereafter, we call $J^{\ominus}(y ; w)$ the minorant of the cost functional.

4.3. Properties of the upper and lower bounds. In this section we show that the estimates (4.4) and (4.6) are exact.

PROPOSITION 4.5.

(i) The majorant $J^{\oplus}(y, u ; \beta ; \tau)$ achieves its exact lower bound on the exact solution $(\bar{y}, \bar{u})$ of Problem P, i.e.,

$$
\inf _{\substack{y \in Y_{0}, \tau \in H_{\text {div }}, u \in \mathcal{U}, \beta>0}} J^{\oplus}(y, u ; \beta ; \tau)=J(\bar{y}, \bar{u}) .
$$

(ii) The minorant $J^{\ominus}(y ; w)$ achieves its exact upper bound on the exact solution $(\bar{y}, \bar{u})$ of Problem P, i.e.,

$$
\sup _{y \in Y_{0}, w \in Y_{0}} J^{\ominus}(y ; w)=J(\bar{y}, \bar{u}) .
$$

Proof. (i) The functional $J^{\oplus}(y, u ; \beta ; \tau)$ provides an upper bound to the cost functional $J(\bar{y}, \bar{u})$ for any $y \in Y_{0}, \tau \in H_{\mathrm{div}}, u \in \mathcal{U}$, and $\beta>0$. Set $u=\bar{u}, y=\bar{y}$ and $\tau=\nabla \bar{y} \in H_{\text {div }}$. Since the pair $(\bar{y}, \bar{u})$ satisfies the system of necessary conditions (2.3)-(2.4), we have

$$
\begin{array}{r}
\|\operatorname{div} \tau+u+f\|=\|\Delta \bar{y}+\bar{u}+f\|=0, \\
\|\tau-\nabla y\|=0, \\
\left.\|u-\xi(y)\|=\| \bar{u}-\left(u^{d}+\frac{1}{a}\left(y^{d}-\bar{y}\right)\right)\right) \|=0 .
\end{array}
$$

Therefore, taking into account statement (b) of Remark 3.1,

$$
J^{\oplus}(\bar{y}, \bar{u} ; \beta ; \nabla \bar{y})=\mathbf{C}_{\bar{y}}=J(\bar{y}, \bar{u}) .
$$

(ii) The functional $J^{\ominus}(y ; w)$ provides a lower bound to the cost functional $J(\bar{y}, \bar{u})$ for any $y \in Y_{0}$ and $w \in Y_{0}$. Put $w=0$ and $y=\bar{y}$. Then

$$
J^{\ominus}(\bar{y} ; 0)=\mathbf{C}_{\bar{y}}=J(\bar{y}, \bar{u}),
$$

which proves the assertion.

5. A posteriori estimates for approximations of the optimal control problem. Here, with the help of previous results, we derive guaranteed upper estimate for the error of the approximate solution of the original optimal control problem. The error is measured in terms of the so-called combined norm:

$$
|[v]|^{2}:=\frac{1}{2}\left\|\nabla y_{v+f}\right\|^{2}+\frac{a}{2}\|v\|^{2}
$$


which is defined for $v \in \mathcal{U}$.

The statement below is the first step in the construction of the estimate.

Proposition 5.1. For any control function $u \in \mathcal{U}$ there holds

$$
|[u-\bar{u}]|^{2}=J\left(y_{u+f}, u\right)-J(\bar{y}, \bar{u}) .
$$

Proof. From (2.4), we know that

$$
\left(\bar{y}-y^{d}\right)+a\left(\bar{u}-u^{d}\right)=0 .
$$

Take an arbitrary function $\phi \in \mathcal{U}$ and multiply (5.1) by $\phi+f$. Integration over $\Omega$ gives

$$
\int_{\Omega}\left(\bar{y}-y^{d}\right)(\phi+f) d x+\int_{\Omega}\left(\bar{u}-u^{d}\right)(\phi+f) d x=0, \quad \forall \phi \in \mathcal{U} .
$$

Let $y_{\phi+f}$ be the exact state for the control $\phi$, i.e.,

$$
\int_{\Omega} \nabla \psi \cdot \nabla y_{\phi+f} d x=\int_{\Omega} \psi(\phi+f) d x, \quad \forall \psi \in Y_{0} .
$$

From (5.2) and (5.3) with $\psi=\bar{y}-y^{d}$ it follows that

$$
\int_{\Omega} \nabla\left(\bar{y}-y^{d}\right) \cdot \nabla y_{\phi} d x+a \int_{\Omega}\left(\bar{u}-u^{d}\right) \phi d x=0 \quad \forall \phi \in \mathcal{U} .
$$

For arbitrary $u \in \mathcal{U}$, we have

$$
\begin{gathered}
J\left(y_{u+f}, u\right)-J(\bar{y}, \bar{u})=\frac{1}{2}\left\|\nabla\left(y_{u+f}-y_{\bar{u}+f}\right)\right\|^{2}+\frac{a}{2}\|u-\bar{u}\|^{2}+ \\
+\int_{\Omega} \nabla\left(\bar{y}-y^{d}\right) \cdot \nabla y_{u-\bar{u}} d x+a \int_{\Omega}\left(\bar{u}-u^{d}\right)(u-\bar{u}) d x .
\end{gathered}
$$

Applying (5.4) with $\phi=u-\bar{u}$, we arrive at the assertion. $\square$

Corollary 5.2. For any $u \in \mathcal{U}$ the upper bound for the error measured in the combined norm is given by

$$
|[u-\bar{u}]|^{2} \leq \mathcal{M}(y, u ; \beta ; \tau, w), \quad \forall \tau \in H_{\mathrm{div}}, w \in Y_{0}, \beta>0,
$$

where

$$
\begin{aligned}
\mathcal{M}(y, u ; \beta ; \tau, w):= & J_{y}^{\oplus}(u ; \beta ; \tau)-J_{y}^{\ominus}(w)= \\
& =\frac{(1+\beta)}{2}\|\tau-\nabla y\|^{2}+\frac{(1+\beta)}{2 \beta} C_{\Omega}^{2}\|\operatorname{div} \tau+u+f\|^{2}+ \\
& +\frac{a}{2}\|u-\xi(y)\|^{2}+\frac{1}{2}\left(\|\nabla w\|^{2}+\frac{1}{a}\|w\|^{2}\right)+ \\
& +\int_{\Omega}(\xi(y)+f) w d x-\int_{\Omega} \nabla w \cdot \nabla y d x .
\end{aligned}
$$


Proposition 5.3. The majorant $\mathcal{M}(y, u ; \beta ; \tau, w)$ attains its exact lower bound on the exact solution of Problem P, i.e.,

$$
\inf _{\substack{y \in Y_{0}, \tau \in H_{\text {div }}, u \in \mathcal{U}, w \in Y_{0}, \beta \in \mathbb{R}_{+}}} \mathcal{M}(y, u ; \beta ; \tau, w)=0 .
$$

Proof. Set $w=0$, then

$$
\begin{array}{r}
\mathcal{M}(y, u ; \beta ; \tau, 0)=\frac{(1+\beta)}{2}\|\tau-\nabla y\|^{2}+\frac{(1+\beta)}{2 \beta} C_{\Omega}^{2}\|\operatorname{div} \tau+u+f\|^{2}+ \\
+\frac{a}{2}\|u-\xi(y)\|^{2} .
\end{array}
$$

Let us write down the system of necessary conditions for the optimal control problem (2.3)-(2.4) including the exact flux $p=\nabla \bar{y}$,

$$
\begin{array}{r}
p=\nabla \bar{y}, \\
\operatorname{div} p+\bar{u}+f=0, \\
\bar{u}=u^{d}+\frac{1}{a}\left(y^{d}-\bar{y}\right) .
\end{array}
$$

The assertion follows if (5.5) we set $\tau=p, y=\bar{y}, u=\bar{u}$, and $\beta=0$.

Remark 7.1 In some situations is is sufficient to use a simplified estimate

$$
|[u-\bar{u}]|^{2} \leq \mathcal{M}(y, u ; \beta ; \tau, 0), \quad \forall \tau \in H_{\mathrm{div}}, \beta>0 .
$$

Setting $w=0$, we reduce the number of auxiliary functions. Moreover, this estimate has a clear sense: it is a weighted sum of penalties for violation of each of the equations in the extended system of necessary conditions (5.6)-(5.8).

6. A posteriori estimate in the full norm. In this section, we show that (5.5) is equivalent to the error measured in the norm

$$
|[v ; q]|_{\lambda}^{2}:=|[v]|^{2}+\frac{1}{2}\|q\|^{2}+\lambda\|\operatorname{div} q\|^{2}, \quad(v, q) \in \mathcal{U} \times H_{\text {div }},
$$

for any $\lambda \in(0, a)$. It is clear that this norm can be viewed as the full primal-dual norm associated with the problem.

Proposition 6.1. There exist constants $C_{\oplus}$ and $C_{\ominus}$ (see (6.3) and (6.4)), such that

$$
C_{\ominus} \mathcal{M}(y, u ; \beta ; \tau, 0) \leq|[(u-\bar{u}) ;(\tau-p)]|_{\lambda}^{2} \leq C_{\oplus} \mathcal{M}(y, u ; \beta ; \tau, 0) .
$$

Proof. Using Young's inequality with $\delta=\frac{1}{2}$, we obtain

$\frac{1}{2}\|\tau-p\|^{2} \leq\left\|\nabla\left(y_{u+f}-\bar{y}\right)\right\|^{2}+\left\|\nabla y_{u+f}-\tau\right\|^{2}=2|[u-\bar{u}]|^{2}-a\|u-\bar{u}\|^{2}+\left\|\nabla y_{u+f}-\tau\right\|^{2}$,

and, therefore, by (5.5) there holds

$$
\frac{1}{2}\|\tau-p\|^{2} \leq 2 \mathcal{M}(y, u ; \beta ; \tau, 0)-a\|u-\bar{u}\|^{2}+\left\|\nabla y_{u+f}-\tau\right\|^{2} .
$$


In addition, for any $\mu>0$ there holds

$$
\lambda\|\operatorname{div} \tau+\bar{u}+f\|^{2} \leq \lambda\left[(1+\mu)\|\operatorname{div} \tau+u+f\|^{2}+\frac{1+\mu}{\mu}\|u-\bar{u}\|^{2}\right] .
$$

Setting $\lambda\left(1+\frac{1}{\mu}\right)=a$ we find that $1+\mu=\frac{a}{a-\lambda}$. The latter relation requires $\lambda \in(0, a)$.

From (6.1) and (6.2) we obtain

$\frac{1}{2}\|\tau-p\|^{2}+\lambda\|\operatorname{div}(\tau-p)\|^{2} \leq 2 \mathcal{M}(y, u ; \beta ; \tau, 0)+\frac{\lambda a}{a-\lambda}\|\operatorname{div} \tau+u+f\|^{2}+\left\|\nabla y_{u+f}-\tau\right\|^{2}$,

which together with (5.5) gives

$$
|[(u-\bar{u}) ;(\tau-p)]|_{\lambda}^{2} \leq 3 \mathcal{M}(y, u ; \beta ; \tau, 0)+\frac{\lambda a}{a-\lambda}\|\operatorname{div} \tau+u+f\|^{2}+\left\|\nabla y_{u+f}-\tau\right\|^{2} .
$$

By Young's inequality with $\delta=\frac{1}{2}$ and the functional majorant for the boundary-value problem we have

$$
\begin{aligned}
\left\|\nabla y_{u+f}-\tau\right\|^{2} & \leq 2\left\|\nabla\left(y_{u+f}-y\right)\right\|^{2}+2\|\nabla y-\tau\|^{2} \leq \\
& \leq 6\|\nabla y-\tau\|^{2}+4 C_{\Omega}^{2}\|\operatorname{div} \tau+u+f\|^{2} .
\end{aligned}
$$

Therefore,

$$
\begin{aligned}
& |[(u-\bar{u}) ;(\tau-p)]|_{\lambda}^{2} \leq \\
& \leq 3 \mathcal{M}(y, u ; \beta ; \tau, 0)+\left(4 C_{\Omega}^{2}+\frac{\lambda a}{a-\lambda}\right)\|\operatorname{div} \tau+u+f\|^{2}+6\|\nabla y-\tau\|^{2} .
\end{aligned}
$$

Recalling the structure of the functional $\mathcal{M}(y, u ; \beta ; \tau, 0)$, we observe that the assertion holds with the constant

$$
C_{\oplus}:=3+\frac{2}{1+\beta} \max \left\{6, \frac{\beta}{C_{\Omega}^{2}}\left(4 C_{\Omega}^{2}+\frac{\lambda a}{a-\lambda}\right)\right\} .
$$

Similar argumentation together with (2.4) gives the lower estimate with

$$
C_{\ominus}=2 \max \left\{2+2 \beta+\frac{c_{1}}{a}, 1+2\left(\frac{c_{1}}{a}\right)^{2}+\frac{c_{2}}{a}, 5+5 \beta+\frac{c_{1}^{2}}{a}, \frac{c_{1}^{2}}{\lambda a}+\frac{c_{2}}{2 \lambda}\right\}^{-1}
$$

where $c_{1}=2 C_{\Omega}^{2}, c_{2}=(1+\beta)\left(8+\beta^{-1}\right) C_{\Omega}^{2}$.

7. Practical implementation. In this section, we discuss main aspects of practical implementation of the estimates (4.4),(4.6), and (5.9).

We restrict ourselves to the case where the problem is solved by usual finite element approximations on a simplicial mesh, which is the same for all functions involved. Let $\mathcal{T}_{h}(\Omega)$ denote a shape-regular simplicial triangulation of $\Omega$. The optimal control problem is solved by means of continuous, piecewise affine finite element approximations for the state $\left(Y_{h} \subset Y_{0}\right)$ and by piecewise constant approximations for the control $\left(\mathcal{U}_{h} \subset \mathcal{U}\right)$. For the free variable $\tau$ we use piecewise quadratic finite element approximations $\left(H_{\text {div }, h} \subset H_{\text {div }}\right)$.

For a given approximate solution of Problem P - $\left(y_{h}, u_{h}\right) \in Y_{h} \times \mathcal{U}_{h}$ - the approach allows to perform two-sided control for the values of the cost functional

$$
J^{\ominus}\left(y_{h} ; 0\right) \leq J\left(y_{u_{h}+f}, u_{h}\right) \leq J^{\oplus}\left(y_{h}, u_{h} ; \beta ; \tau\right), \quad \forall \tau \in H_{\mathrm{div}}, \beta>0
$$


and simultaneously control the error of the approximate solution

$$
\left|\left[u_{h}-u\right]\right|^{2} \leq \mathcal{M}\left(y_{h}, u_{h} ; \beta ; \tau, 0\right), \quad \forall \tau \in H_{\text {div }}, \beta>0 .
$$

The auxiliary function $\tau$ and parameter $\beta$ can be selected once and used for both estimates. In the results exposed below a suitable substitution for $\tau$ was found by minimization of the majorant $\mathcal{M}\left(y_{h}, u_{h} ; \beta ; \tau_{h}, 0\right)$ w.r.t. $\beta>0$ and $\tau_{h} \in H_{\mathrm{div}, h}$. We denote the minimizers by $\bar{\beta}$ and $\bar{\tau}_{h}$.

Example. In this example, the data are as follows: $\Omega=(0,1)^{2}, a=1, y^{d}\left(x_{1}, x_{2}\right)=$ $0, u^{d}\left(x_{1}, x_{2}\right)=-1+\sin \left(\frac{\pi x_{1}}{2}\right)+\sin \left(\frac{\pi x_{2}}{2}\right), f\left(x_{1}, x_{2}\right)=2 \pi^{2} z\left(x_{1}, x_{2}\right)-\min \left\{u^{d}\left(x_{1}, x_{2}\right)-\right.$ $\left.z\left(x_{1}, x_{2}\right), 0\right\}, z\left(x_{1}, x_{2}\right)=\sin \left(\pi x_{1}\right) \sin \left(\pi x_{2}\right)$. The exact solution is known and given by $\bar{y}\left(x_{1}, x_{2}\right)=z\left(x_{1}, x_{2}\right), \bar{u}\left(x_{1}, x_{2}\right)=u^{d}\left(x_{1}, x_{2}\right)-z\left(x_{1}, x_{2}\right), J(\bar{y}, \bar{u})=2.5924$.

To estimate the quality of the error control we use the numbers

$$
J^{\oplus}:=J^{\oplus}\left(y_{h}, u_{h} ; \bar{\beta} ; \bar{\tau}_{h}\right) \quad \text { and } \quad J^{\ominus}:=J^{\ominus}\left(y_{h} ; 0\right)
$$

that show the quality of the a posteriori control with respect to the cost functional. The quantities

$$
\theta_{y}=\frac{\left\|y_{h}-y(u)\right\|_{H^{1}}}{\|y(u)\|_{H^{1}}} 100 \%, \quad \theta_{u}=\frac{\left\|u_{h}-u\right\|}{\|u\|} 100 \%
$$

show the relative errors in the state and control respectively. The efficiency index

$$
I=\sqrt{\frac{\mathcal{M}\left(y_{h}, u_{h} ; \bar{\beta} ; \bar{\tau}_{h}, 0\right)}{\left|\left[u_{h}-u\right]\right|^{2}}}
$$

characterizes the quality of the error control in terms of the combined norm. Its value is always greater than 1 .

In Table 1, we present the results obtained on various uniform meshes. By $N_{h}$ we denote the number of nodes in the current mesh. Together with the relative errors in the state and the control, we show the results of the a posteriori control of the solution in terms of the quantities $J^{\oplus}, J^{\ominus}$, and $I$.

Table 1.

\begin{tabular}{c||c|c||c|c||c}
\hline$N_{h}$ & $\theta_{y}, \%$ & $\theta_{u}, \%$ & $J^{\ominus}$ & $J^{\oplus}$ & $I$ \\
\hline 25 & 36.99 & 31.61 & 2.2378 & 2.6752 & 1.0962 \\
81 & 19.01 & 14.68 & 2.4990 & 2.6055 & 1.0541 \\
289 & 9.62 & 7.18 & 2.5687 & 2.5952 & 1.0441 \\
1089 & 4.91 & 3.62 & 2.5865 & 2.5931 & 1.0419 \\
4225 & 2.64 & 1.91 & 2.5909 & 2.5926 & 1.0415 \\
\hline
\end{tabular}

This results confirm the efficiency of the estimates w.r.t. both, the value of the cost functional and the error measured in the combined norm. 


\section{REFERENCES}

[1] M. Ainsworth and J.T. Oden, A posteriori error estimation in finite element analysis. Wiley, New York, 2000.

[2] I. Babuška and W.C. Rheinboldt, A posteriori error estimates for the finite element method. Int. J. Numer. Meth. Engrg. (1978), No. 12, 1597-1615.

[3] I. Babuška and W.C. Rheinboldt, Error estimates for adaptive finite element computations. SIAM J. Numer. Anal. (1978), No. 15(4), 736-754.

[4] I. Babuška and T. Strouboulis, The finite element method and its reliability, Clarendon Press, Oxford, 2001.

[5] W. Bangerth, R. Rannacher. Adaptive finite element methods for differential equations. Birkhäuser, Berlin, 2003.

[6] R. E. Bank, A. Weiser. Some a posteriori error estimators for elliptic partial differential equations. Math. Comp. 44 (1985), No. 170, 283-301.

[7] R. Beck, R. Hiptmair, R.H.W. Hoppe, and B. Wohlmuth, Residual based a posteriori error estimators for eddy current computation. M2AN Math. Modelling and Numer. Anal. 34, 159-182 (2000).

[8] R. Becker, H. Kapp, and R. Rannacher. Adaptive finite element methods for optimal control of partial differential equations: Basic concept, SIAM J. Control Optim. 39, 113-132, 2000.

[9] R. Becker, R. Rannacher. An optimal control approach to error estimation and mesh adaptation in finite element methods. In: Acta Numerica (A. Iserles, ed.), 10: 1-102, Cambridge University Press, 2001.

[10] C. Carstensen and S. Bartels, Each averaging technique yields reliable a posteriori error control in FEM on unstructured grids. I: Low order conforming, nonconforming, an mixed FEM, Math. Comp., 71 (2002), 239, 945-969.

[11] C. Carstensen and R.H.W. Hoppe, Error reduction and convergence for an adaptive mixed finite element method, to appear in Math. Comp.

[12] C. Carstensen and R.H.W. Hoppe, Convergence analysis of an adaptive nonconforming finite element method, submitted to Numer. Math.

[13] C. Carstensen and R.H.W. Hoppe, Convergence analysis of an adaptive edge finite element method for the $2 \mathrm{~d}$ eddy current equations, J. Numer. Math. 13, (2005).

[14] C. Carstensen, R. Verfürth. Edge residuals dominate a posteriori error estimates for low order finite element methods. SIAM J. Numer. Anal. 36 (1999), No. 5, 1571-1587.

[15] W. Dörfler, A convergent adaptive algorithm for Poisson's equation, SIAM J. Numer. Anal. 33, (1996), 1106-1124.

[16] Eriksson, K. and Estep, D. and Hansbo, P. and Johnson, C. Computational Differential Equations. Cambridge University Press, Cambridge, 1995

[17] A. Gaevskaya, R.H.W. Hoppe, and S. Repin. A Posteriori Estimates for Cost Functionals of Optimal Control Problems. to appear in Proc. ENUMATH 2005 Conf., Santiago de Compostela, July 18-22, 2005.

[18] M. Hintermüller, R.H.W. Hoppe, Y. Iliash, and M. Kieweg. An adaptive finite element method for a distributed elliptic control problem with control constraints. submitted

[19] R.H.W. Hoppe, and B. Wohlmuth. Adaptive multilevel techniques for mixed finite element discretizations of elliptic boundary value problems. SIAM J. Numer. Anal. 34 (1997), 16581681

[20] C. Johnson, P. Hansbo. Adaptive finite element methods in computational mechanics. Comput. Methods Appl. Mech. Engrg. 101 (1992), 143-181.

[21] R. Li, W. Liu, H. Ma, and T. Tang. Adaptive finite element approximation for distributed optimal control problems. SIAM J. Control Optim. Vol. 41, No. 5 (2002), 1321-1349

[22] J. L. Lions. Optimal Control of Systems Governed by Partial Differential Equations. Springer, Berlin-Heidelberg-New York. 1971.

[23] W. Liu and N. Yan. A posteriori error estimates for distributed optimal control problems. Adv. Comp. Math. 15, 285-309, 2001.

[24] W. Liu and N. Yan. A posteriori error estimates for optimal control problems governed by parabolic equations, Numer. Math. 93, 497-521, 2003.

[25] W. Liu and N. Yan. A posteriori error estimates for convex boundary control problems. Preprint, Institute of Mathematics and Statistics, University of Kent, Canterbury, 2003.

[26] P. Morin, R.H. Nochetto, and K.G. Siebert, Data oscillation and convergence of adaptive FEM, SIAM J. Numer. Anal., 38, (2000), 466-488.

[27] P. Morin, R.H. Nochetto, and K.G. Siebert, Local problems on stars: a posteriori error estimators, convergence, and performance, Math. Comp. 72, (2003), 1067-1097.

[28] P. Neittaanmäki and S. Repin. Reliable methods for computer simulation. Error control and a 
posteriori estimates. Elsevier, New York, 2004.

[29] S. Repin, A posteriori error estimation for nonlinear variational problems by duality theory, Zapiski Nauchn. Semin. POMI,243 (1997), 201-214.

[30] S. Repin, A posteriori error estimation for variational problems with uniformly convex functionals, Math. Comp., 69 (2000), 230, 481500.

[31] S. Repin, Two-Sided Estimates of Deviation from Exact Solutions of Uniformly Elliptic Equations, Amer. Math. Soc. Transl., (2) Vol. 209 (2003), 143-171.

[32] R. Verfürth, A review of a posteriori error estimation and adaptive mesh-refinement techniques, Wiley and Sons, Teubner, New York, 1996.

[33] L.B. Wahlbin, Superconvergence in Galerkin finite element methods, Lecture Notes in Mathematics 1605, Springer-Verlag, 1995.

[34] O.C. Zienkiewicz and J. Z. Zhu, A simple error estimator and adaptive procedure for practical engineering analysis, Internat. J. Numer. Meth. Engrg., 24 (1987), 337-357.

[35] O.C. Zienkiewicz and J. Z. Zhu, Adaptive techniques in the finite element method, Commun. Appl. Numer. Methods, 4 (1988), 197-204. 